\title{
Pathogenic variants identified using whole-exome sequencing in Chinese patients with primary ciliary dyskinesia
}

Yutian Ye

Shenzhen People's Hospital

Qijun Huang

Shenzhen People's Hospital

Lipeng Chen

Shenzhen People's Hospital

Chunxian Liang

Shenzhen People's Hospital

Kaixue Zhuang

Shenzhen People's Hospital

Tao Liu

Shenzhen People's Hospital

Fang Yuan

Shenzhen People's Hospital

Xiangxia Zhang

Shenzhen People's Hospital

Jie Li

Shenzhen People's Hospital

Hua Dang

Shenzhen People's Hospital

Rongchang Chen

Shenzhen People's Hospital

Yingyun Fu ( $\square$ yingyunfu2017@163.com )

Shenzhen People's Hospital

Yongjian Yue ( $\nabla$ yueyongj@163.com )

Shenzhen People's Hospital https://orcid.org/0000-0003-4184-0025

Research

Keywords: primary ciliary dyskinesia, DNAAF3, DNAI1, whole-exome sequencing

Posted Date: March 2nd, 2021

DOI: https://doi.org/10.21203/rs.3.rs-156279/v2

License: (c) (1) This work is licensed under a Creative Commons Attribution 4.0 International License. Read Full License 


\section{Abstract \\ Background}

Primary ciliary dyskinesia (PCD) is an autosomal recessive disorder. The genetic factors contributing to PCD pathogenesis remain elusive for approximately $20-35 \%$ of patients with complex and abnormal clinical phenotypes. Our study aimed to identify causative variants of sporadic PCD genes using whole-exome sequencing (WES).

\section{Result}

All patients were diagnosed with PCD based on clinical phenotype or transmission electron microscopy (TEM) images of cilia. WES and bioinformatic analysis were then conducted for patients with PCD. Identified candidate variants were validated by Sanger sequencing. Pathogenicity of candidate variants was then evaluated using in silico software and the American College of Medical Genetics and Genomics (ACMG) database. In total, 15 rare variants were identified in five patients with PCD. Five new variants of CCDC40, DNAH1, DNAAF3, and DNA/1 were considered causative variants and included one splicing and three homozygous variants.

\section{Conclusion}

Our study demonstrated that patients with PCD carry rare causative variants of multiple genes. Our findings indicated that not only known causative genes but also other functional genes should be considered for heterogeneous genetic disorders.

\section{Background}

Primary ciliary dyskinesia (PCD) is an autosomal recessive disorder characterized by respiratory distress, tympanitis, sinusitis, and bronchiectasis [1]. Kartagener syndrome (MIM\# 244400) is a subtype of PCD exhibiting the situs inversus phenotype. The estimated prevalence of PCD ranges from 1 in 10,000 to 1 in 15,000 among Europeans [2]. PCD is diagnosed by measuring nasal nitric oxide and through brush biopsy [3]. Transmission electron microscopy (TEM) is used to obtain a definitive diagnosis by visualizing the ultrastructure of cilia. Male PCD patients present with infertility due to dysfunctional immotile or dyskinetic sperm flagella [4].

Genetic screening has improved our understanding of the pathogenesis of inherited PCD [5]. Previous genetic and functional studies revealed a series of PCD-associated genes causing congenital ultrastructural abnormalities resulting in dysfunctional immotile cilia [6]. Inherited genetic variations are present in approximately $60 \%$ of $\mathrm{PCD}$ cases, indicating a high genetic heterogeneity of this disorder [3]. Mutations in inner dynein arm (IDA) and outer dynein arm (ODA) complex-coding genes cause ciliary abnormalities, but some patients with confirmed PCD clinical features also show normal ciliary motility and ultrastructure [7]. Interestingly, some studies have reported that PCD with heterotaxy and airway ciliary dysfunction only involves heterozygous mutations in PCD-associated genes [8]. Another study showed that an autosomal dominant mutation in FOXJ1 can also cause a distinct motile ciliopathy related to defective ciliogenesis, presenting a similar clinical phenotype as that of PCD [9]. These findings suggest the potential involvement of unknown genes and mechanisms in pathologies associated with ciliary function.

Next generation sequencing has advanced the identification of candidate causative genes in PCD [10]. Here, we aimed to identify pathogenic gene variants for sporadic PCD in Chinese patients. Genetic testing combined with TEM and clinical feature analysis have greatly improved the definitive diagnosis of and identification of genetic defects associated with PCD. These tools will allow us to identify risk factors for siblings of individuals with PCD, facilitating the genetic diagnosis of and counseling for this disorder.

\section{Methods}

\section{Subjects and PCD diagnosis}

Five PCD patients, together with control individuals, were enrolled in the study from 2008 to 2020 . Patients were diagnosed with PCD according to the classic characterization and guidelines [11]. The control subjects included in the study were the healthy siblings or parents of patients and unrelated healthy individuals. Ethical approval was obtained from the ethics committee of the Shenzhen People's Hospital (Guangdong, China), as per the ethical standards recommended in the 1964 Helsinki Declaration of Helsinki. Informed consent was obtained from each subjects enrolled in the study. High-resolution computed tomography (CT), magnetic resonance (MR) imaging, and TEM examination were conducted to investigate chronic sinusitis, bronchiectasis, situs inversus, and ciliary ultrastructural defects.

Identification of variants by wholeexome sequencing (WES) and bioinformatic analysis

Page 2/9 
Genomes from all subjects were sequenced using the NovaSeq platform (Illumina). Variants revealed using WES data were analyzed using the Genome Analysis Toolkit and Annovar [12]. Subsequently, all variants were filtered based on minor allele frequency (MAF<0.1\%), using the following databases: 1000 genomes, CG69, and gnomAD (http://gnomad.broadinstitute.org/). Sorting Intolerant From Tolerant, MutationTaster, Polymorphism Phenotyping v2 (PolyPhen-2), and Combined Annotation Dependent Depletion were used to assess the pathogenicity of variants. Further, an in-house filtering pipeline was used to identify candidate variants.

\section{Pathogenicity assessment and validation of candidate variants}

Gene function- and frequency-based filtering analyses were conducted for all variants. In total, 46 pathogenic genes highly associated with PCD were selected as candidate gene variants, as described previously [13]. Variant pathogenicity assessment was conducted using bioinformatic software and ACMG [14]. The classification and interpretation of variants followed the ACMG guidelines. Identified candidate causative variants were validated in patients with PCD and control subjects using the Sanger sequencing platform. The Chromas software was used to analyze the sequencing results (Technelysium Pty Ltd.).

\section{PCR and sequencing confirmation of the CCDC40 splicing variant}

Total RNA was extracted from whole blood of the PCD patients and reverse-transcribed using the RR036A kit (TAKARA, Japan). Primers for CCDC40 exons were designed using the IDT PrimerQuest tool (https://sg.idtdna.com/Primerquest/Home/Index). cDNA segments transcribed from exon CCDC40 mRNA were amplified using standard PCR. The primers for exon 13 to exon 14 transcripts were as follows: primer 4 F- 5区 GGACCAGGACGTGAAGAAAG 3囚, and R-5区 CTGTGTCACCTTGACCATCTC; and for exon 14 to exon 15 transcripts: primer 6 F- 5》 GATCGACGAGCACGATGG 3区, and R-GAGCTTCTTCAGGTCGTTGT 3®. Transcript products of the splicing variant in patient KT8 were confirmed by Sanger sequencing and agarose gel electrophoresis.

\section{Results}

\section{Demographics and clinical phenotypes of PCD patients}

PCD patients were diagnosed using typical methods, as described in the Materials and Methods section. All patients with PCD had the clinical characteristics of recurrent cough and expectoration for several years (Table 1). Chest CT or MR imaging revealed bronchiectasis in all patients, and situs inversus in four patients (Fig. 1). The four subjects with situs inversus had kartagener syndrome. TEM examination showed the presence of ODA and IDA defects as well as microtubular disorganization of the cilia in KT7 and KT8 (Fig. 1).

Table 1

Clinical characteristics of PCD patients

\begin{tabular}{|lllllll|}
\hline PCD ID & Age & Gender & Recurrent cough & Situs inversus & Bronchiectasis & Bronchitis \\
\hline KT6 & 30 & Female & Yes & Yes & Yes & Yes \\
KT7 & 32 & Female & Yes & Yes & Yes & Yes \\
\hline KT8 & 48 & Male & Yes & Yes & Yes & Yes \\
KT9 & 48 & Female & Yes & Yes & Yes & No \\
KT10 & 12 & Male & Yes & No & Yes & Yes \\
\hline
\end{tabular}

\section{Identification of rare variants by bioinformatic analysis}

To identify candidate PCD-causative gene variants in the five Chinese PCD patients, we filtered variants to obtain rare ones based on function and frequency. In total, we identified 15 variants of candidate genes with MAF $<1 \%$ in the East Asian population from the 1000 genomes, ExAC, and gnomAD databases (Table 2). Six novel variants were identified for five genes that were not recorded in the gnomAD and dbSNP databases. We confirmed one homozygous variant of DNAI1 and two homozygous variants of CCDC40 in three patients with PCD to be likely genetic factors contributing to the disorder (Table 2). No other rare heterozygotes were found together with the identified potential pathogenic heterozygous variant in KT6 and KT7, indicating that the genetic defects of these patients were not caused by compound heterozygotes. 
Table 2

The interpretation and pathogenicity assessment of candidate variants

\begin{tabular}{|c|c|c|c|c|c|c|c|}
\hline Chr & Start & refGene & Alteration & $\begin{array}{l}\text { PCD } \\
\text { Carrier }\end{array}$ & Variant type & $\begin{array}{l}\text { gnomeAD } \\
\text { EAS }\end{array}$ & $\begin{array}{l}\text { ACMG } \\
\text { Classification }\end{array}$ \\
\hline chr7 & 21781653 & DNAH11 & NM_001277115:exon49:c.A8023G:p.12675V & KT6 & Missense & 0.0006 & $\begin{array}{l}\text { PM1 + PP5 + } \\
\text { BS1 + BP4 }\end{array}$ \\
\hline chr19 & 55672134 & DNAAF3 & NM_001256714:exon9:c.G1126T:p.E376X & KT7 & Stop-gain & NA & $\begin{array}{l}\text { PVS1 + PM2 } \\
+ \text { PP3 }\end{array}$ \\
\hline chr17 & 40086999 & TTC25 & UNKNOWN & KT7 & Missense & NA & $\mathrm{PM} 1+\mathrm{BP} 4$ \\
\hline chr3 & 52392666 & DNAH1 & NM_015512:exon25:c.C4179A:p.N1393K & KT7 & Missense & NA & $\mathrm{PM} 1+\mathrm{BP} 4$ \\
\hline chr5 & 13911508 & DNAH5 & NM_001369:exon12:c.C1631T:p.T544I & KT8 & Missense & 0.0093 & $\begin{array}{l}\text { PM1 + PP5 + } \\
\text { BS1 + BP4 }\end{array}$ \\
\hline chr3 & 52388915 & DNAH1 & NM_015512:exon21:c.C3537G:p.Y1179X & KT8 & Stop-gain & NA & $\begin{array}{l}\text { PVS1 + PM2 } \\
+ \text { PP3 }\end{array}$ \\
\hline chr2 & 84861727 & DNAH6 & NM_001370:exon30:c.C4615G:p.Q1539E & KT8 & Missense & 0.0019 & PM1 \\
\hline chr14 & 50100519 & DNAAF2 & NM_001083908:exon1:c.G1349T:p.S450I & KT8 & Missense & 0.0006 & BP4 \\
\hline chr17 & 78059800 & CCDC40 & NM_001243342:exon14:c.2236-2A>- & KT8 & $\begin{array}{l}\text { Homozygote } \\
\text { Splicing }\end{array}$ & NA & \\
\hline chr9 & 34506853 & DNAl1 & NM_001281428:exon13:c.A1304C:p.H435P & KT9 & $\begin{array}{l}\text { Homozygote } \\
\text { Missense }\end{array}$ & NA & $\begin{array}{l}\text { PM1 + PM2 + } \\
\text { PP3 }\end{array}$ \\
\hline chr17 & 11556249 & DNAH9 & NM_001372:exon14:c.G2525A:p.R842Q & KT9 & Missense & NA & NA \\
\hline chr3 & 50382932 & ZMYND10 & NM_001308379:exon1:c.A79G:p.M27V & KT9 & Missense & 0.0012 & PM2 \\
\hline chr17 & 78023917 & CCDC40 & NM_001243342:exon7:c.994dupT:p.Y332fs & KT10 & $\begin{array}{l}\text { Homozygote } \\
\text { frameshift } \\
\text { insertion }\end{array}$ & NA & \\
\hline chr7 & 21726746 & DNAH11 & NM_001277115:exon33:c.A5651G:p.H1884R & KT10 & Missense & NA & $\mathrm{PM} 1+\mathrm{PM} 2$ \\
\hline chr3 & 52430923 & DNAH1 & NM_015512:exon73:c.C11650T:p.R3884C & KT10 & Missense & NA & PM1 \\
\hline
\end{tabular}

\section{ACMG guidelines based pathogenicity assessment of candidate variants}

Candidate variants were assessed using in silico software and ACMG guidelines (Table 2). All potential causative variants were validated by Sanger sequencing (Fig. 2). The frameshift insertion in CCDC4O (c.994dupT) is a pathogenic homozygous variation that was identified as the genetic cause of PCD in KT10. Another homozygous variation in DNA/1 was present in KT9 in the absence of other rare pathogenic gene variants, the DNA/7 variant could be the potential cause of $P C D$, even though it is considered as a VUS according to the ACMG criteria. Further evidence is needed to confirm the association between this homozygous variation in DNA/1 and the PCD phenotype. Two stop-gain heterozygous variants of DNAAF3 and DNAH1 were identified in KT7 and KT8. We did not find other rare variants of these two genes, indicating that PCD in the two cases was not only caused by heterozygous but also by trans-heterozygous gene interactions.

\section{Confirmation of the pathogenicity of the CCDC40 splicing variant}

The homozygous deletion c.2236-2A>- in CCDC40 (NM_017950) found in KT8 alters the nucleotide sequence at the splicing site near exon 14. To confirm whether this variation causes abnormal mRNA transcription, CDNA sequencing was conducted in KT8 and normal control. Genomic DNA sequencing revealed the splicing deletion in KT8 (Fig. 3A). Further PCR and sequencing results showed that the portion of the cDNA between exon 13 and exon 14 was lost in the variant containing the CCDC40 splicing deletion in KT8 (Fig. 3C), while the portion of CDNA was normally present in the control. CDNA-based PCR sequencing showed normal CCDC4O CDNA products in the normal control (Fig. 3B). This indicated that the splicing deletion variant of $C C D C 40$ (c.2236-2A>-) resulted in the production of a shorter mRNA transcript compared with normal control. This finding, together with the clinical phenotype resulting from the $C C D C 40$ defect suggest that splicing variant c.2236-2A>- is likely to be pathogenic.

No rare pathogenic variants were identified in KT6, except for a likely benign rare variant in DNAH1. Two rare variants were predicted as likely benign, and the others were classified as VUS (Table 2). These variants were considered to not be associated with PCD based on the ACMG 
guidelines. In summary, five rare variants were considered to potentially be causative for PCD based on ACMG guidelines (Table 2).

\section{Discussion}

In the present study, WES was conducted to identify candidate PCD-causative gene variants in five patients. Five causative variations were considered pathogenic in CCDC40, DNAH1, DNAAF3, and DNA/1. Three homozygous variants, including splicing variants, were identified for $C C D C 40$ and $D N A / 1$. Further analyses showed that the splicing variant of $C C D C 40$ might result in the production of a shortened protein, which may contribute to PCD pathogenesis.

To date, there has been strong functional evidence for the contribution of over 50 genes to PCD pathogenesis, with the genes mainly causing structural abnormalities of motile cilia, such as complete or partial deletion of IDAs or ODAs [6]. Defects in CCDC40 are responsible for IDA defects and microtubular disorganization [15]. Our study identified two homozygous variants of CCDC40. The carrier of these variants showed the classic PCD phenotype involving situs inversus and bronchiectasis. TEM examination demonstrated that the abnormal structure of cilia in the carrier was consistent with genetic defects in CCDC4O. Our subsequent analysis confirmed that a splicing deletion in $C C D C 40$ could shorten its mRNA transcript. Based on this evidence, the splicing deletion variant of $C C D C 40$ may likely be a PCD-causative variant; The exact functions and mechanisms of the splicing variant of CCDC4O in PCD pathogenesis need to be confirmed through further studies.

One novel PCD-causative homozygous variant was identified in DNA/1. ACMG predicted this variant to be a VUS, but the variant was predicted to be pathogenic by all in silico softwares. DNA/1 encodes the intermediate chain of ODAs [16]. Mutations in DNA/1 result in situs inversus and primary ciliary dyskinesia [17]. The carrier of the DNA/1 variant exhibited the classic PCD phenotype and had no other rare gene variant. We speculated that the $D N A / 1$ variant is the main genetic factor contributing to PCD in this case. Therefore, appropriate functional studies would be helpful to confirm the pathogenicity of the DNA/1 variant. We found a novel stop-gain heterozygous variant of DNAAF3 but no other pathogenic variants in one of the assessed PCD patients. The mutation in DNAAF3 causes ODA and IDA defects in cilia [18]. Two rare VUS mutations of DNAH1 and TTC25 were also identified, but whether the observed trans-heterozygous interactions between these mutations could play a role in PCD remains to be confirmed. Identification of ciliary structural defects is helpful to confirm the association between a gene mutation and PCD phenotype. Unfortunately, some patients in our study refused to undergo TEM examination.

As PCD is an autosomal recessive disorder, homozygous or compound heterozygous mutations are commonly considered contributing genetic factors. However, our present and previous studies demonstrated that heterozygous pathogenic mutations are frequent in patients with PCD [13], especially frameshift insertions or deletions and stop-gain or -loss mutations. None of the other candidate pathogenic variants were present together. Interestingly, the inheritance model of the candidate variants is very similar to that for autosomal dominant disorders. Some studies have shown that autosomal dominant variants can also cause defects in ciliogenesis with a similar clinical phenotype as that of PCD [9]. However, further investigation is required to determine whether these heterozygous variants can cause PCD on their own.

\section{Conclusion}

In conclusion, our study identified five candidate PCD-causative gene variants of CCDC40, DNAH1, DNAAF3, and DNA/1. More evidence is required to confirm the pathogenicity of various types of genetic variants in PCD, since PCD is a heterogeneous genetic disorders. Confirmation of the causative genetic factors and clinical ciliary defect type in PCD is an important step toward developing personalized clinical diagnosis and genetic counseling.

\section{Declarations}

\section{Ethics approval and consent to participate}

The project was approved by the ethics committee of the Ethics Committee of Shenzhen People's Hospital. All procedures performed in studies involving human participants in accordance with the 1964 Declaration of Helsinki ethical standards.

\section{Consent for publication}

Written Informed consent for publication was obtained from all participants, all of the co-author approved the publish of the article.

\section{Availability of data and materials}

The datasets used or analysed during the current study are available from the database of GSA-Human (https://bigd.big.ac.cn/). 


\section{Competing interests}

The authors declare that the research was conducted in the absence of any commercial or financial relationships that could be construed as a potential conflict of interest.

\section{Funding}

The study was supported by the Guangdong Provincial Natural Science Foundation (2018A030310674), the Guangdong Provincial Science and Technology Project (2017A020214016), the Key Laboratory of Shenzhen Respiratory Diseases (ZDSYS201504301616234), and Shenzhen Science and Technology Project (JCYJ20170413093032806).

\section{Authors' contributions}

Yongjian Yue and Yingyun Fu prepared the project proposal and study design. Yongjian Yue and Lipeng Chen conducted bioinformatics and statistical analysis of sequencing data. Qijun Huang, Fang Yuan, Chunxian Liang, and Kaixue Zhuang conducted sample collection and Sanger sequencing validation. Qijun Huang, Xiangxia Zhang, and Yutian Ye conducted clinical diagnosis of PCD. Tao Liu, Jie Li and Rongchang Chen assisted with the prepared and revised manuscript. All the authors have read and approved the final manuscript.

\section{Acknowledgments}

Thanks to laboratory support from the Key Laboratory of Shenzhen Respiratory Diseases.

\section{References}

1. Horani A, Ferkol TW. Advances in the Genetics of Primary Ciliary Dyskinesia: Clinical Implications. Chest. 2018;154(3):645-52.

2. Bush A, Chodhari R, Collins N, Copeland F, Hall P, Harcourt J, et al. Primary ciliary dyskinesia: current state of the art. Arch Dis Child. 2007;92(12):1136-40.

3. Lucas JS, Paff T, Goggin P, Haarman E. Diagnostic Methods in Primary Ciliary Dyskinesia. Paediatr Respir Rev. 2016;18:8-17.

4. Liu C, Miyata H, Gao Y, Sha Y, Tang S, Xu Z, et al. Bi-allelic DNAH8 Variants Lead to Multiple Morphological Abnormalities of the Sperm Flagella and Primary Male Infertility. Am J Hum Genet. 2020;107(2):330-41.

5. Khalid F, Hannah WB, Gaston BM. Rapid Advances in Primary Ciliary Dyskinesia Research. A Brief Update for Pulmonologists. Am J Respir Crit Care Med. 2019;199(2):136-8.

6. Lucas JS, Davis SD, Omran H, Shoemark A. Primary ciliary dyskinesia in the genomics age. Lancet Respir Med. 2020;8(2):202-16.

7. Wallmeier J, Al-Mutairi DA, Chen CT, Loges NT, Pennekamp P, Menchen T, et al. Mutations in CCNO result in congenital mucociliary clearance disorder with reduced generation of multiple motile cilia. Nat Genet. 2014;46(6):646-51.

8. Wilson CW, Nguyen CT, Chen MH, Yang JH, Gacayan R, Huang J, et al. Fused has evolved divergent roles in vertebrate Hedgehog signalling and motile ciliogenesis. Nature. 2009;459(7243):98-102.

9. Wallmeier J, Frank D, Shoemark A, Nothe-Menchen T, Cindric S, Olbrich H, et al. De Novo Mutations in FOXJ1 Result in a Motile Ciliopathy with Hydrocephalus and Randomization of Left/Right Body Asymmetry. Am J Hum Genet. 2019;105(5):1030-9.

10. Fassad MR, Patel MP, Shoemark A, Cullup T, Hayward J, Dixon M, et al. Clinical utility of NGS diagnosis and disease stratification in a multiethnic primary ciliary dyskinesia cohort. J Med Genet. 2020;57(5):322-30.

11. Shapiro AJ, Davis SD, Polineni D, Manion M, Rosenfeld M, Dell SD, et al. Diagnosis of Primary Ciliary Dyskinesia. An Official American Thoracic Society Clinical Practice Guideline. Am J Respir Crit Care Med. 2018;197(12):e24-39.

12. Wang K, Li M, Hakonarson H. ANNOVAR: functional annotation of genetic variants from high-throughput sequencing data. Nucleic Acids Res. 2010;38(16):e164.

13. Yue Y, Huang Q, Zhu P, Zhao P, Tan X, Liu S, et al. Identification of Pathogenic Mutations and Investigation of the NOTCH Pathway Activation in Kartagener Syndrome. Frontiers in genetics. 2019;10:749.

14. Richards S, Aziz N, Bale S, Bick D, Das S, Gastier-Foster J, et al. Standards and guidelines for the interpretation of sequence variants: a joint consensus recommendation of the American College of Medical Genetics and Genomics and the Association for Molecular Pathology. Genet Med. 2015;17(5):405-24. 
15. Becker-Heck A, Zohn IE, Okabe N, Pollock A, Lenhart KB, Sullivan-Brown J, et al. The coiled-coil domain containing protein CCDC40 is essential for motile cilia function and left-right axis formation. Nat Genet. 2011;43(1):79-84.

16. Zariwala MA, Leigh MW, Ceppa F, Kennedy MP, Noone PG, Carson JL, et al. Mutations of DNAI1 in primary ciliary dyskinesia: evidence of founder effect in a common mutation. Am J Respir Crit Care Med. 2006;174(8):858-66.

17. Guichard C, Harricane MC, Lafitte JJ, Godard P, Zaegel M, Tack V, et al. Axonemal dynein intermediate-chain gene (DNAl1) mutations result in situs inversus and primary ciliary dyskinesia (Kartagener syndrome). Am J Hum Genet. 2001;68(4):1030-5.

18. Mitchison HM, Schmidts M, Loges NT, Freshour J, Dritsoula A, Hirst RA, et al. Mutations in axonemal dynein assembly factor DNAAF3 cause primary ciliary dyskinesia. Nat Genet. 2012;44(4):381-9, S1-2.

\section{Figures}

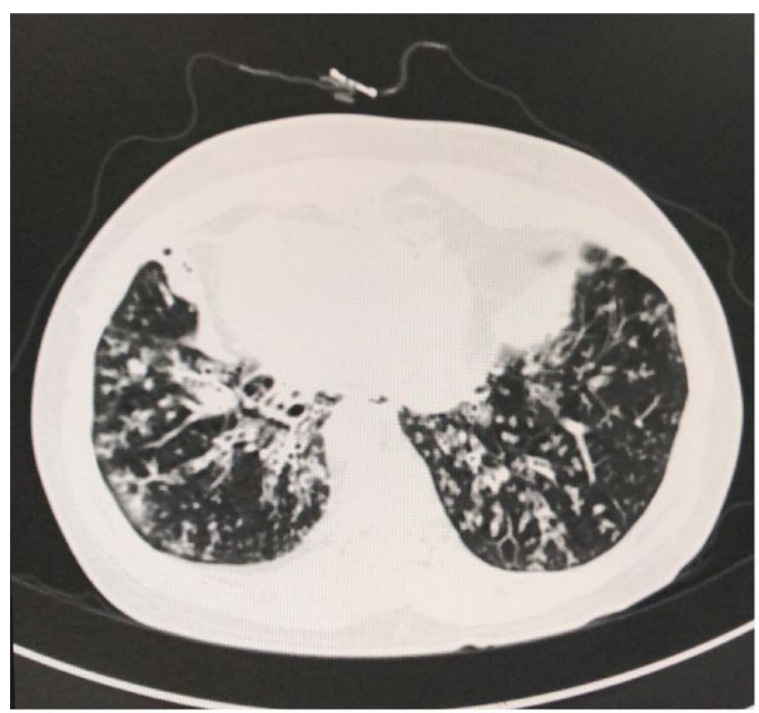

a

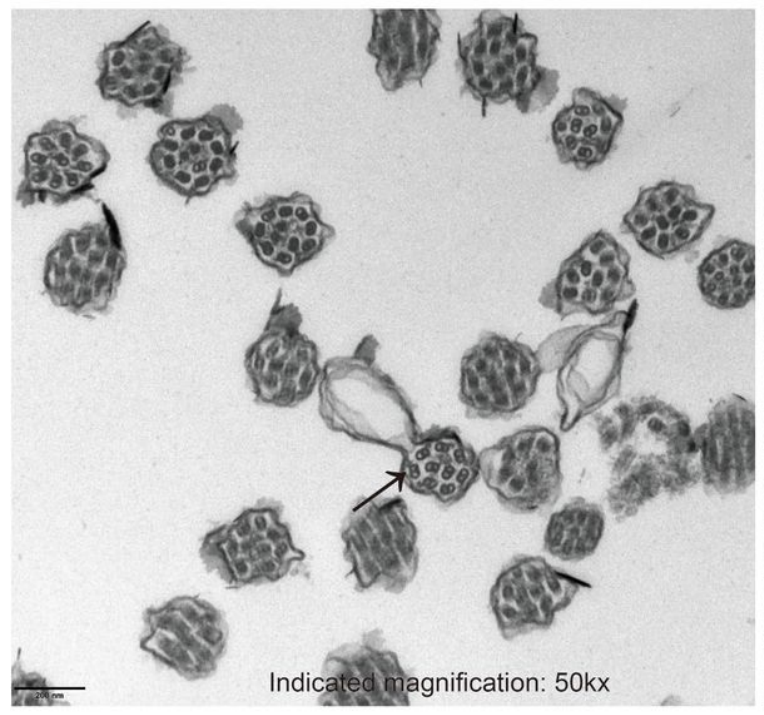

C

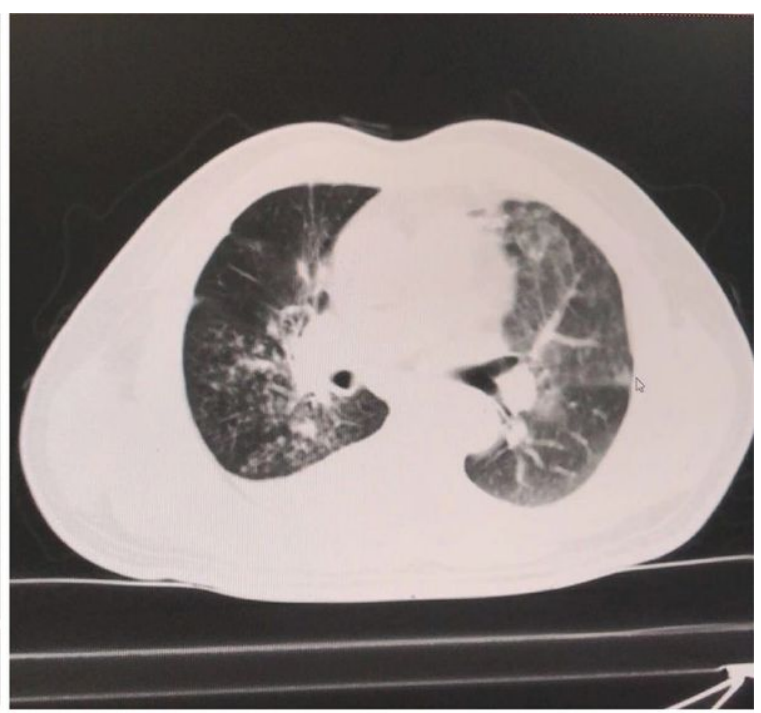

b

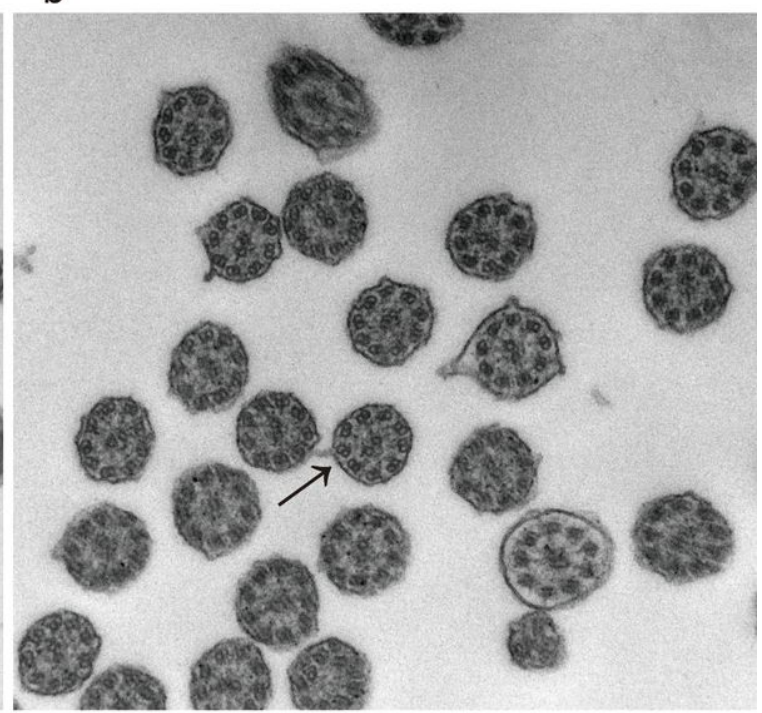

d

\section{Figure 1}

PCD diagnosis using traditional methods. Chest images showed bronchiectasis and situs inversus in KT9 (a) and bronchiectasis in KT10 (b). TEM examination showed structural defects in KT7 (c) and KT8 (d) cilia, the arrows represent the ODA, IDA defects and microtubular disorganization. 


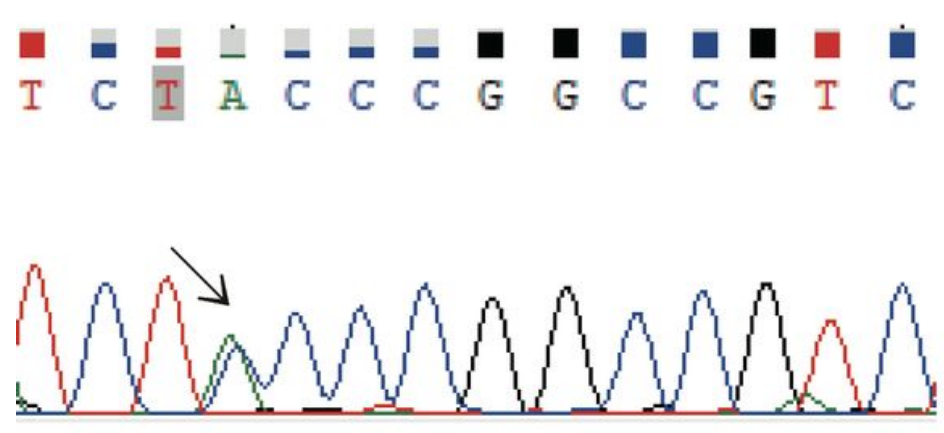

KT7 DNAAF3 c.G1126T

$\begin{array}{llllllllllllll}\mathbf{G} & \mathrm{C} & \mathrm{C} & \mathrm{C} & \mathrm{T} & \mathrm{C} & \mathrm{A} & \mathrm{G} & \mathrm{A} & \mathrm{C} & \mathrm{C} & \mathrm{C} & \mathrm{T}\end{array}$

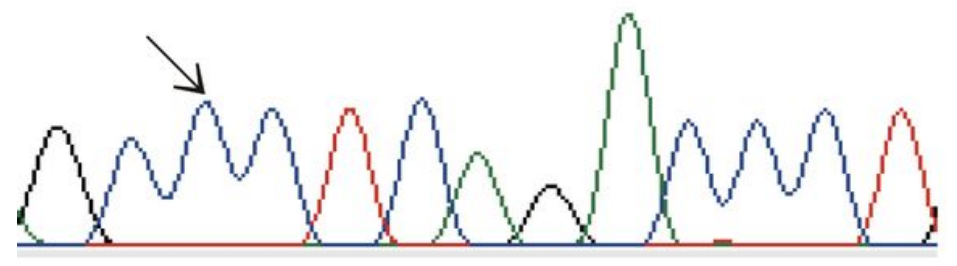

KT9 DNAI1 c.A1304C

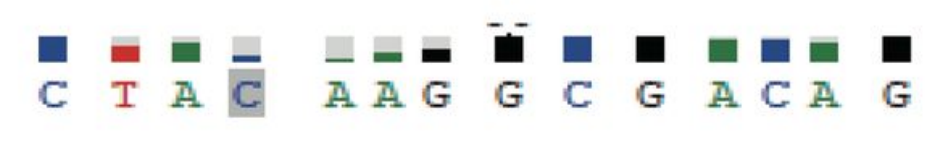

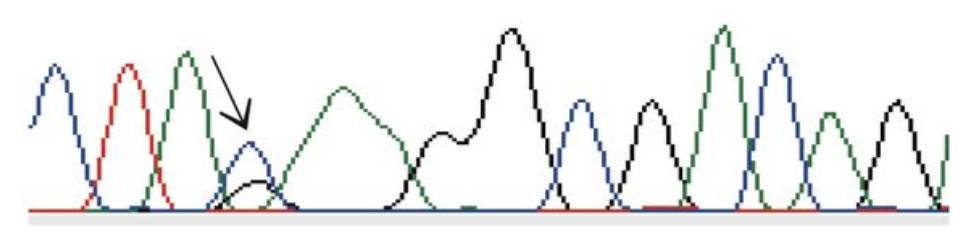

KT8 DNAH1 c.C3537G

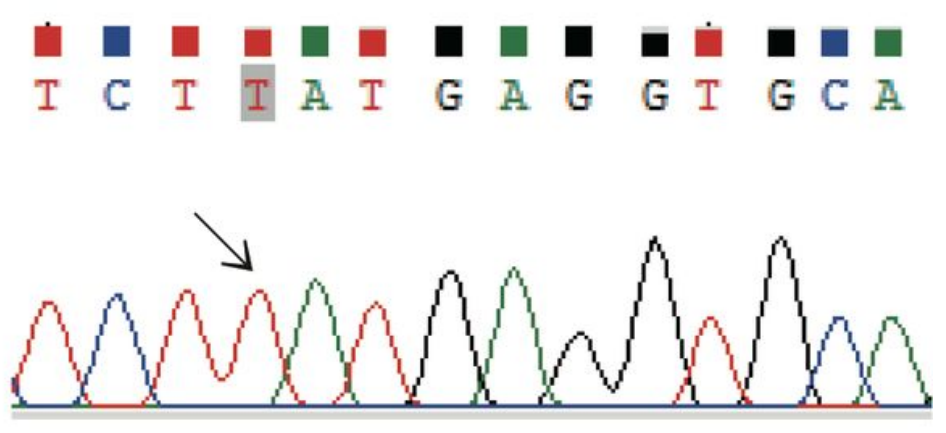

KT10 CCDC40 c.994dupT

Figure 2

Validation of causative candidate variants by Sanger sequencing.

Tर

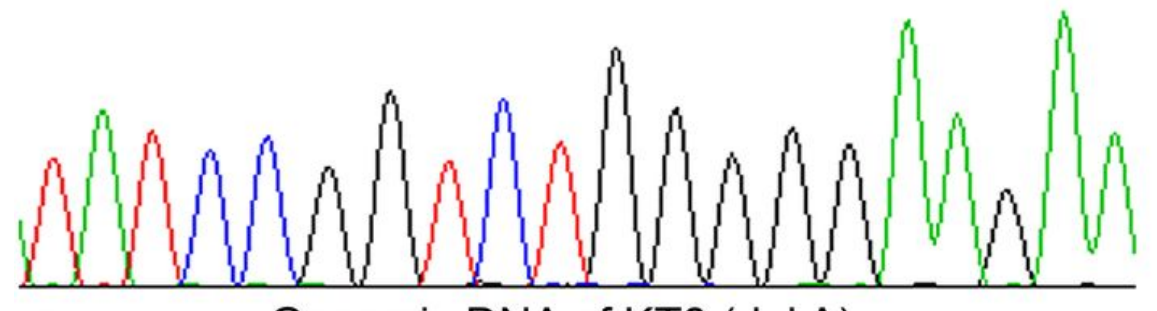

a Genomic DNA of KT8 $(\operatorname{del} A)$

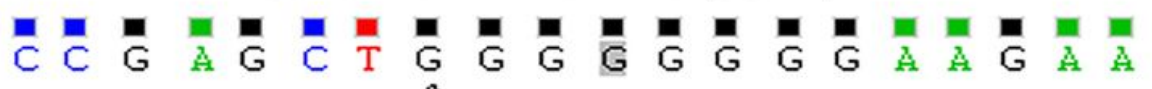

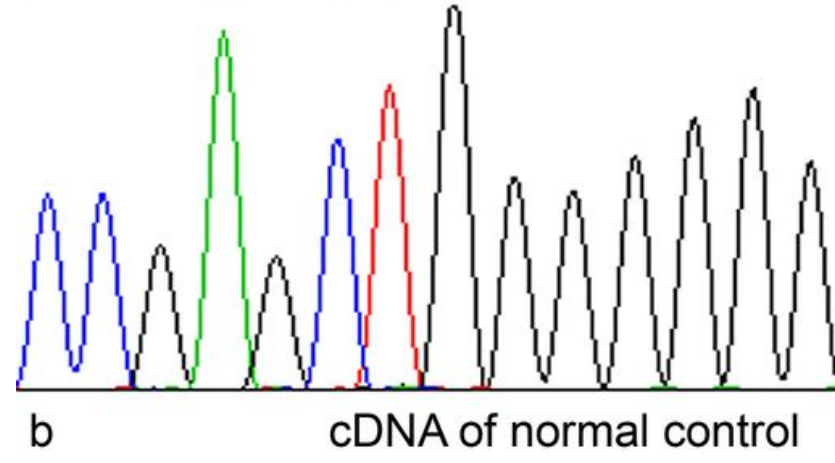

KT8 Con KT8 Con 
Figure 3

(a) Splicing deletion in the KT8 genomic DNA sequence. (b) cDNA sequencing of the control showed a normal sequence between exon 13 and exon 14 of the transcript. (c) Agarose gel electrophoresis showed the CCDC40 CDNA PCR products was lost in KT8. 